\title{
A Universal Approach towards Light-Responsive Two-Dimensional Electronics: Chemically Tailored Hybrid van der Waals Heterostructures
}

Yuda Zhao', Simone Bertolazzi', Paolo Samorì

${ }^{1}$ University of Strasbourg, CNRS, ISIS UMR 7006, 8 allée Gaspard Monge, F-67000

Strasbourg, France

KEYWORDS

two-dimensional materials, photochromic molecules, dielectric, optoelectronic devices, diode

\section{ABSTRACT}

Stimuli responsive hybrid van der Waals heterostructures (vdWHs), composed of organic molecular switches superimposed on inorganic 2D materials (2DMs), can combine the outstanding physical properties of the latter components with the virtually-infinite variety of tunable functionality of molecules, thereby offering an efficient protocol for the development of highperformance multifunctional materials and devices. The use of light as remote control to modulate the properties of semiconducting 2DMs when interfaced with photochromic molecules, suffers from both the limitation associated to the persistent photoconductivity characterizing the 2DMs 
and the finite thermal stability of the photochromic molecule in its different states. Here we have devised a universal approach towards the fabrication of optically switchable electronic devices comprising a few nanometer thick azobenzene (AZO) layer physisorbed on 2D semiconductors supported on trap-free polymer dielectric. The joint effect of the improved 2D/dielectric interface, the molecule's light-modulated dipolar doping, and the high thermal stability of cis-AZO offers highest control over the reversible and efficient charge carrier tuning in 2D semiconductors with a preserved high performance in $2 \mathrm{D}$ FET, as quantified in terms of carrier mobility and $\mathrm{I}_{\mathrm{on}} / \mathrm{I}_{\text {off }}$ ratio. The device has the potential to operate as optical memory with 4 current levels and long retention time ( $>15$ hours). Furthermore, by using CMOS-compatible micropatterning process, the photoswitchable resistor-diode transition has been achieved on hybrid lateral heterojunction devices. Our approach is of general applicability towards the generation of high-performance hybrid vdWHs for the emergence of functional and responsive devices.

The physical superposition of dissimilar two-dimensional materials (2DMs), which are free of dangling-bond on their surfaces, offers numerous solutions towards the development of van der Waals heterostructures (vdWHs) with unusual properties and functions. ${ }^{1-5}$ Alongside 2D-2D vdWHs, the tailoring of hybrid organic/inorganic vdWHs provides another feasible way for nanoscale material integration by also offering the possibility to introduce the desired functionality through the molecular design. ${ }^{6,7}$ Molecules can by design be capable of responding to external stimuli: they can change their state, even in a reversible manner, when subjected to external inputs like photons, magnetic fields, $\mathrm{pH}$ changes, etc. $^{8}$ In such a way, they can controllably switch between two or more (meta-)stable states exhibiting diverse physical properties (intrinsic dipole 
moment, energy level, electron affinity). Among them, photochromic molecules are widely studied compounds, which can respond to light-stimuli at specific wavelengths. ${ }^{9,10}$ Due to their extremely high surface to volume ratio, the atomically-thin 2DMs are very sensitive to the influence of the surrounding environment (for both the top and bottom surface of 2DMs), and their properties (such as, photoluminescence (PL) and electronic charge transport) can thereof be easily modified by external inputs. ${ }^{11-14}$ The photochromic molecules can directly interact with 2DMs by modulating their properties (including band alignment and charge-carrier concentration) as a response to light stimuli. Since their interaction is ruled by weak forces thus determining a weak coupling, the hybrid vdWHs, consisting of 2DMs and molecular switches, can retain the impressive electrical characteristics of $2 \mathrm{D}$ crystals and enrich the advanced functionality provided by the switchable molecules. Therefore, the combination of $2 \mathrm{DMs}$ with molecular systems represents a viable protocol to develop multifunctional hybrid materials and devices suitable for advanced logic, memory and sensing applications. ${ }^{15,16}$

Previous work was mostly focused on the hybrid system based on graphene (or reduced graphene oxide) and photochromic molecules. ${ }^{17-19}$ However, due to the lack of bandgap in graphene, the prospects for its electronic application remain problematic. To the best of our knowledge, only a few reports have been published on hybrid vdWHs based on 2D semiconductors and photochromics, ${ }^{20,21}$ especially for the applications in the light-tunable field effect transistors (FETs) and $p-n$ diodes. This is because the existence of persistent photoconductivity (PPC) effect after light irradiation in 2D FETs greatly prolongs the device response time and even hinders the observation of the reversible doping effect brought into play by the physisorbed molecules. ${ }^{22,23}$ Alongside, some molecules require long irradiation time to undergo isomerization and display a modest thermal stability. ${ }^{24}$ Such characteristics makes hybrid vdWHs based on 2D semiconductors 
and photochromic molecules not suitable for responsive devices. It is therefore essential to develop a universal approach to suppress the PPC effect and modulate via light stimuli the carrier concentration of 2D layers in a controllable manner, with the ultimate goal of realizing the $2 \mathrm{D}$ multiresponsive thus multifunctional devices.

In this work, we fabricated hybrid vdWHs formed from photochromic molecules (azobenzene, $\mathrm{AZO})$ and 2D semiconductors $\left(\mathrm{MoS}_{2}\right.$ or black phosphorus (BP)). The $\mathrm{AZO} / \mathrm{MoS}_{2}$ hybrid system shows light-tunable Raman and PL spectra of monolayer $\mathrm{MoS}_{2}$ induced by the switchable doping from AZO molecules. In order to minimize the influence from PPC effect, a trap-free polymer dielectric has been exploited in the optoelectronic devices. The photoresponse speed of monolayer $\mathrm{MoS}_{2}$ FET on polymer dielectric is 3 times faster than that on $\mathrm{SiO}_{2}$. The light-controlled charge transport in $\mathrm{MoS}_{2}$ and BP FETs has been realized via reversible photochemical isomerization of the AZO layers. Through the tuning of the population ratio of trans-/cis-AZO molecules it was possible to set four conductance states in $\mathrm{MoS}_{2}$ FET. Significantly, the retention time for metastable cis-AZO state exceeds 15 hours, largely surpassing current state of the art in AZO based memory devices (corresponding to about 2.7 hours). ${ }^{25}$ By taking advantage of the reversible doping capacity of AZO on ambipolar BP FETs, in combination with standard micropatterning process, we have fabricated BP based lateral heterojunctions to achieve the local control of the doping level in different regions of the channel and realize the light-tunable $p$ - $n$ diodes. These results provide evidence for the huge technological potential possessed by hybrid vdWHs for the emergence of high-performance multifunctional optoelectronic devices including opticallycontrolled transistors, multi-bit optical memories, and light-tunable diodes.

\section{Results and Discussion}


Photoswitching of the AZO/MoS 2 hybrid system. As prototypical light-addressable molecule for the functionalization of $2 \mathrm{D}$ semiconductors we have focused our attention to a commercial alkoxy-substituted AZO molecule, i.e. 4-(decyloxy) azobenzene. Its special design comprises a photoresponsive AZO unit grafted at the end of a long alkoxy side chain, the latter being capable of promoting efficient physisorption on the surface of $2 \mathrm{D}$ semiconductors to form functional superlattices. ${ }^{21,26}$ Compared with other photochromic molecules, AZO molecules show several advantages, such as large conformational changes, sizable change in dipole moment, low rate of photobleaching, and robust isomerization. In fact, spiropyran/merocyanine are prone to undergo photodegradation and the equilibrium of isomerization is greatly affected by the solvent type (and related $\mathrm{pH}){ }^{24}$ The different dipole moment of the AZO in its trans and cis form makes such molecule a suitable model system to explore the reversible light-modulated doping/undoping of 2DMs when interfaced. ${ }^{17,27}$ The electric field generated by molecular dipoles, analogous to that of an external gate, can modulate the carrier concentration of 2D semiconductors. This dipolar interaction appears being a more universal physical process compared to charge transfer (which requires the band alignment) in diarylethene containing hybrids and devices thereof. ${ }^{28}$

Figure 1a portrays the scheme of the hybrid vdWHs based on photochromic molecules and 2D semiconductor. When exposed to light at specific wavelengths, the AZO molecules can undergo efficient isomerization between different states exhibiting diverse dipole moment. The carrier concentration and even carrier polarity of 2D semiconductors can therefore be directly modulated via dipolar interactions with the switching photochromic molecules. For 4-(decyloxy) azobenzene molecules, theoretical calculations also predict that the contribution to the work function (WF) shift of hybrid AZO/2D system from the charge transfer is smaller than that from the dipolar interaction. ${ }^{27}$ Hence, the dipolar interaction plays the dominant role in the doping of $2 \mathrm{D}$ 
semiconductors by 4-(decyloxy) azobenzene molecules. The external light stimuli act as the remote control to induce modification in the electronics of the $2 \mathrm{D}$ semiconductors, which can be finally read out by monitoring changes detected by optical spectroscopy and electrical measurements. The reversible photoisomerization of AZO molecules on quartz substrates has been demonstrated by UV-Vis absorption, as displayed in Supporting Information Figure S1. In order to characterize the surface morphology of 4-(decyloxy) azobenzene absorbed on monolayer $\mathrm{MoS}_{2}$ we used atomic force microscopy (AFM) in intermittent contact mode. The topographical images of pristine monolayer $\mathrm{MoS}_{2}$ on $\mathrm{SiO}_{2}$ is displayed in Figure 1b. It reveals smooth surface having a thickness of $\sim 0.78 \mathrm{~nm}$. The AZO molecules are solubilized in ethanol and spin-coated onto the surface of monolayer $\mathrm{MoS}_{2}$. After the deposition of the molecules, the roughness on the $\mathrm{MoS}_{2}$ terraces is enhanced due to the molecular overlayer (Figure 1c). The flake thickness also raises up to $\sim 4.06 \mathrm{~nm}$, indicating a thickness of the 4-(decyloxy) azobenzene layer corresponding to $\sim 3.28$ $\mathrm{nm}$. The phase images reveal that the molecules prefer to assemble onto the $\mathrm{MoS}_{2}$ surface rather than onto $\mathrm{SiO}_{2}$ substrate. The AFM images of $\mathrm{AZO}$ molecules on $\mathrm{SiO}_{2}$ (Figure 1d) show random aggregates with the thickness of $\sim 3.17 \mathrm{~nm}$.

Raman and PL spectroscopy are versatile tools to characterize the correlation between the crystallinity and the electronic structure, enabling also to unravel the effect of various perturbations on the electronics of $2 \mathrm{DMs}$, such as defect, strain and doping. ${ }^{29-31}$ Therefore, the electronic interaction between switching molecules and $\mathrm{MoS}_{2}$ can be directly monitored by the spectroscopic study. Figure 2a shows the Raman spectra of pristine $\mathrm{MoS}_{2}$ and $\mathrm{MoS}_{2}$ with physisorbed 4(decyloxy) azobenzene molecules (AZO/MoS 2 ). The two main Raman-active phonon modes, $A_{1 \mathrm{~g}}$ and $E_{2 \mathrm{~g}}^{1}$, can be observed with $<20 \mathrm{~cm}^{-1}$ wavenumber difference, which infers the monolayer nature of the flake. ${ }^{32}$ Compared with pristine $\mathrm{MoS}_{2}$, the physisorption of trans-AZO only induces 
slight change of Raman peaks, such as the broadening of $A_{1 \mathrm{~g}}$ peak. After switching molecules from trans to $c i s$ form, we can observe the blue-shift of $A_{1 \mathrm{~g}}$ mode by $1.06 \mathrm{~cm}^{-1}$, the peak sharpening of $A_{1 \mathrm{~g}}$ mode by $0.64 \mathrm{~cm}^{-1}(\Delta \mathrm{FWHM})$ and the almost unchanged $E_{2 \mathrm{~g}}^{1}$ mode. The $A_{1 \mathrm{~g}}$ mode is more sensitive to the carrier doping due to a stronger electron-phonon coupling and the $E_{2 \mathrm{~g}}^{1}$ mode is sensitive to strain due to the symmetry breaking. ${ }^{33,}{ }^{34}$ Therefore, cis-AZO molecules induce $p$ doping effect on $\mathrm{MoS}_{2}$ and the molecules are physisorbed on $\mathrm{MoS}_{2}$ surface without change of $\mathrm{MoS}_{2}$ crystal structure. The doping concentration by cis-AZO can be estimated from the $A_{1 \mathrm{~g}}$ peak shift, which is $\sim 2.3 \times 10^{12} \mathrm{~cm}^{-2} .{ }^{33}$ Figure $2 \mathrm{~b}$ shows the $\mathrm{PL}$ spectra of pristine $\mathrm{MoS}_{2}$ and AZO/MoS hybrid system. The A exciton of monolayer $\mathrm{MoS}_{2}$ consists of both neutral excitons $(E(\mathrm{X}) \approx 1.88$ $\mathrm{eV}$ ) and negatively charged excitons (trions, $E\left(\mathrm{X}^{-}\right) \approx 1.84 \mathrm{eV}$ ), so that the trion spectral weight depends on the amount of doping in the $2 \mathrm{D}$ semiconducting sheet. ${ }^{35}$ Based on the peak fitting results in Figure 2b, the spectra weight of trion in the pristine sample is higher than that of exciton, due to the $n$-type doping typically occurring in $\mathrm{MoS}_{2}$ crystals. After the physisorption of transAZO molecules, the weight percentage of the trion peak increases and the exciton peak almost vanishes, demonstrating a slight increase of electron density in $\mathrm{MoS}_{2}$. After irradiation with UV light, the PL peak blue shifts and the intensity increases. The fitting curves reveal that the trion spectral weight decreases and the neutral exciton peak dominates the spectra. The PL results are consistent with Raman results, demonstrating the depletion of electrons in $\mathrm{MoS}_{2}$ by cis-AZO. After irradiation with visible light, both the Raman and PL spectra convert back to the trans-AZO/MoS 2 condition. By and large, the spectroscopic study provides unambiguous evidence of the efficient light-controlled reversible doping of $\mathrm{MoS}_{2}$ determined by the photoresponsive $\mathrm{AZO}$ adlayer. From another point of view, it also demonstrates the possibility to reversibly control the light emission (wavelength, intensity) of $\mathrm{MoS}_{2}$ monolayer by the $\mathrm{AZO}$ molecules. 
Improved photoresponse dynamics by using trap-free polymer dielectric. When interfacing photoswitches with 2D semiconductors and using the hybrid system for light-responsive optoelectronic applications, the presence of the PPC effect represents a major drawback towards the efficient light-triggered device operation. ${ }^{23}$ The PPC effect has been mainly attributed to the trap states at the interface and results in a slow (long) response speed (time). ${ }^{22,36}$ To achieve fast response speed and low interfacial trap density, in our optoelectronic devices we opted to use an appropriate hydroxyl-free gate dielectric, i.e. divinyltetramethyldisiloxane-bis(benzocyclobutene) $(\mathrm{BCB}),{ }^{37}$ in order to optimize the $2 \mathrm{D} /$ dielectric interface, improve the photoresponse dynamics, and minimize the PPC effect. Compared with other commonly used trap-free dielectric in 2D electronics, such as poly(methyl methacrylate) (PMMA) and hexagonal boron nitride (h-BN), ${ }^{38,39}$ $\mathrm{BCB}$ dielectric shows simple and large-scale fabrication process, low processing temperature, high thermal stability, and excellent compatibility with the conventional microfabrication process. Such a thin BCB layer can be deposited on the arbitrary substrate as a universal solution to engineer the dielectric surface. The $\mathrm{BCB}$ monomers are spin-coated onto $\mathrm{SiO}_{2} / \mathrm{Si}$ substrate and annealed to achieve optimal crosslinking (BCB thickness $\sim 60 \mathrm{~nm}$ ). Figure 3a compares the PL spectra of monolayer $\mathrm{MoS}_{2}$ on $\mathrm{BCB}$ and on $\mathrm{SiO}_{2}$ substrate, respectively. When compared with neat $\mathrm{SiO}_{2}$, the presence of a BCB overlayer determines on the PL of $\mathrm{MoS}_{2}$ both a blue shift and a notable increase in intensity. The $\mathrm{SiO}_{2}$ dielectric with surface dangling bonds and impurities have been demonstrated to introduce unintentional $n$-type doping on $\mathrm{MoS}_{2} .{ }^{40}$ The negatively charged excitons dominate the PL peak and the high density of interface trap states at $\mathrm{SiO}_{2}$ surface strongly quenched the PL emission. In contrast, BCB substrate enhances the PL emission and shows less 
$n$-doping effect. The PL peak is dominated by the neutral excitons on BCB, which is similar to the PL emission of suspended $\mathrm{MoS}_{2}$ flake. ${ }^{41}$

Back-gated $\mathrm{MoS}_{2}$ FETs were fabricated on both $\mathrm{BCB}$ and $\mathrm{SiO}_{2}$ substrate with patterned top $\mathrm{Au}$ electrodes (channel length/width is $0.7 \mu \mathrm{m} / 2.4 \mu \mathrm{m}$ and $0.6 \mu \mathrm{m} / 1.2 \mu \mathrm{m}$ for device on $\mathrm{BCB}$ and $\mathrm{SiO}_{2}$ substrate, respectively). Figure $3 \mathrm{~b}$ shows the $2 \mathrm{D}$ conductivity $\left(\sigma=\frac{I_{d}}{V_{d}} \frac{L}{W}\right)$ of monolayer $\mathrm{MoS}_{2} \mathrm{FET}$ as a function of back-gated voltage $\left(V_{\mathrm{g}}\right)$, where $I_{\mathrm{d}}$ is the source-drain current, $V_{\mathrm{d}}$ is the source-drain voltage and $L / W$ is the length/width ratio of the channel. At $V_{\mathrm{g}}=0 \mathrm{~V}$, the conductivity of monolayer $\mathrm{MoS}_{2}$ on $\mathrm{SiO}_{2}$ substrate is 4 times greater than that on BCB substrate and the threshold voltage $V_{\text {th }}$ of $\mathrm{MoS}_{2} \mathrm{FET}$ on BCB substrate shifts to a more positive value ( $\Delta V_{\mathrm{th}} \approx 20 \mathrm{~V}$ compared to that on $\mathrm{SiO}_{2}$ substrate). We can extract the electron concentration $\left(n_{\mathrm{e}}\right)$ of $\mathrm{MoS}_{2}$ on both substrate, which is $1.34 \times 10^{12} \mathrm{~cm}^{-2}$ and $0.85 \times 10^{12} \mathrm{~cm}^{-2}$ on $\mathrm{SiO}_{2}$ and on BCB surface, respectively. Significantly, the electrical transport results on the substrate-induced doping are consistent with those obtained from the spectroscopic measurements. The hysteresis test of $\mathrm{MoS}_{2}$ FET (Figure 3c and 3d, the value decreases from $7.3 \mathrm{~V}$ on $\mathrm{SiO}_{2}$ substrate to $4.1 \mathrm{~V}$ on $\mathrm{BCB}$ substrate) further demonstrates the lower trap density at the $\mathrm{MoS}_{2} / \mathrm{BCB}$ interface $\left(2.86 \times 10^{12} \mathrm{~cm}^{-2} \mathrm{eV}^{-1}\right)$ compared to the case of the $\mathrm{MoS}_{2} / \mathrm{SiO}_{2}$ interface $\left(4.69 \times 10^{12} \mathrm{~cm}^{-2} \mathrm{eV}^{-1}\right)$.

Due to the high quality of the $\mathrm{MoS}_{2}$ when interfaces with $\mathrm{BCB}$ coated $\mathrm{SiO}_{2}$, one great advantage induced by $\mathrm{BCB}$ substrate consists in the enhanced photoresponse dynamics of the $\mathrm{MoS}_{2}$ photodetector and the minimization of PPC effect. Figure $3 \mathrm{e}$ shows the time-resolved photoresponse of the $\mathrm{MoS}_{2}$ FET under $530 \mathrm{~nm}$ light on both substrates without gate bias. The device on BCB substrate displays a steep photocurrent rise and decay when switching on and off the illumination. The photocurrent reaches saturation quite fast on BCB substrate, while the 
photocurrent continuously increases during the $20 \mathrm{~s}$ light illumination on $\mathrm{SiO}_{2}$ substrate. This can be ascribed to the present of trap states either at the $\mathrm{MoS}_{2} / \mathrm{SiO}_{2}$ interface or in the $\mathrm{MoS}_{2}$ itself. The photo-excited free carriers can be trapped in these states and thereby hindering the recombination of the electron-hole pair. The long-lifetime carriers continuously increase the photocurrent. In addition, the slow relaxation time of the trapped carriers results in the apparent PPC effect of $\mathrm{MoS}_{2}$ FET on $\mathrm{SiO}_{2}$ substrate (Figure 3e, top). We also measure the transfer curves before and after light illumination (Supporting Information Figure S2). The results show that after illumination, the threshold voltage shifts and the current greatly increases for the $\mathrm{MoS}_{2} \mathrm{FET}$ on $\mathrm{SiO}_{2}$ substrate. All these negative effects will inhibit the observation of switchable doping of $\mathrm{MoS}_{2}$ by photochromic molecules. In contrast, the time-resolved photoresponse curve (Figure 3e, bottom) and the transfer curve (Figure 3f) of $\mathrm{MoS}_{2} \mathrm{FET}$ on $\mathrm{BCB}$ coated $\mathrm{SiO}_{2}$ after light illumination demonstrate that the PPC effect is markedly reduced on BCB. A direct comparison of the response dynamic is displayed in Supporting Information Figure S3. The presence of trap states can dramatically influence the dynamics of the $\mathrm{MoS}_{2}$ photodetector. The rise $\left(\tau_{\mathrm{r}}\right)$ and decay $\left(\tau_{\mathrm{d}}\right)$ times (defined as the time taken by the current to change from $10 \%$ to $90 \%$ of its maximum) of $\mathrm{MoS}_{2}$ device on $\mathrm{BCB}$ is $2.60 \mathrm{~s}$ and $8.88 \mathrm{~s}$, respectively, which is $\sim 3$ times smaller than that on neat $\mathrm{SiO}_{2}(9.73 \mathrm{~s}$ and $22.04 \mathrm{~s}$, respectively). Within the time range of the measurement, the current of $\mathrm{MoS}_{2} \mathrm{FET}$ on $\mathrm{SiO}_{2}$ is still larger than pristine level. Therefore, the actual decay time of $\mathrm{MoS}_{2}$ device on $\mathrm{SiO}_{2}$ is longer. The field-effect mobility of $\mathrm{MoS}_{2}$ on BCB substrate $\left(11.97 \mathrm{~cm}^{2} \mathrm{~V}^{-1} \mathrm{~s}^{-1}\right)$ is higher than that on $\mathrm{SiO}_{2}$ substrate $\left(10.30 \mathrm{~cm}^{2} \mathrm{~V}^{-1} \mathrm{~s}^{-1}\right)$ due to the suppressed scattering effect. ${ }^{42,43}$ By and large, the use of $\mathrm{BCB}$ dielectric represents a powerful method to minimize the PPC effect and improve the photoresponse dynamics for functional devices. 
Photoswitchable electrical transport in $\mathrm{AZO} / \mathrm{MoS}_{2}$ hybrid devices. After optimization of $\mathrm{MoS}_{2} /$ dielectric interface by employing a thin BCB interlayer, we characterize the switching behavior of the hybrid AZO/MoS 2 FET under light control. The schematic of the device structure is shown in Figure 4a. The AZO molecules are physically adsorbed on the top surface of $\mathrm{MoS}_{2}$ layer by the same spin-coating process described above and the thickness $(\sim 3.3 \mathrm{~nm})$ has been determined by AFM. Compared with $\mathrm{MoS}_{2}$ without molecular adsorption, the trans-AZO molecules induce $n$-type doping effect on $\mathrm{MoS}_{2}$ (Figure S4). Figure $4 \mathrm{~b}$ and $4 \mathrm{c}$ display a representative switching cycle of monolayer $\mathrm{MoS}_{2}$ FET with AZO functionalization. After UV irradiation, a decrease of drain current in the transfer curves (Figure $4 \mathrm{~b}$ ) can be observed under the whole range of the gate bias and the threshold voltage shifts towards the positive direction $\left(\Delta V_{\text {th }}\right.$ $=18.48 \mathrm{~V}$, compared with trans-AZO/MoS 2 FET). Therefore, cis-AZO molecules deplete the electron carriers and induce $p$-type doping in $\mathrm{MoS}_{2}$. The carrier concentration change induced by the isomerization of AZO from trans to cis form is $\sim 1.11 \times 10^{12} \mathrm{~cm}^{-2}$. The drain current decreases $\sim 34 \%$ at $V_{\mathrm{g}}=60 \mathrm{~V}$ and a large current ratio $\left(I_{\text {trans }} / I_{\text {cis }} \approx 450\right)$ is achieved at $V_{\mathrm{g}}=-38 \mathrm{~V}$ due to the shift of the threshold voltage. The determined field-effect electron mobility slightly depends on the state of the AZO: when the latter is in its trans form it amounts to $13.86 \mathrm{~cm}^{2} \mathrm{~V}^{-1} \mathrm{~s}^{-1}$ whereas upon isomerization to cis it decreases down to $10.89 \mathrm{~cm}^{2} \mathrm{~V}^{-1} \mathrm{~s}^{-1}$ ( $22 \%$ reduction). The reverse process from the low conductance state to the high conductance state can be triggered by visible light irradiation. By doing this, the drain current and the threshold voltage get restored to the original values. Interestingly, the output curves in Figure 4c show a linear relationship under low drain biases $\left(V_{\mathrm{d}}<0.1 \mathrm{~V}\right)$ regardless of the isomerization of AZO. Such a finding demonstrates a low contact resistance and the molecules has negligible effect on the contact area. 
Figure $4 \mathrm{~d}$ displays the time evolution of the drain current response for one switching cycle (20-s rest in the dark, 10-s UV light, 20-s rest in the dark, 50-s visible light and 20-s rest in the dark). A current increase and drop can be observed suddenly when the light is turned on and off. It is generally attributed to the generation and relaxation of photoexcited carriers. Under UV irradiation, the current gradually decreases due to the trans-cis isomerization of AZO and under visible light irradiation, the current gradually increases because of the transition back of AZO from cis to trans form. The rate constants for the trans-cis isomerization can be extracted from the timedependent photoresponse curves (Supporting Information Figure S5) and the results are comparable with that in literature. ${ }^{17}$ Supporting Information Figure S6 shows the photoresponse of the hybrid device for seven switching cycles. It clearly exhibits two resistance state corresponding to the doping of $\mathrm{MoS}_{2}$ by trans and cis form of AZO molecules, respectively. And after over 50-cycle switching tests in two months, the device preserved the switching ability with a little deterioration. To rule out potential artifacts, we have performed control experiments on bare $\mathrm{MoS}_{2}$ FET (Supporting Information Figure S7). During irradiation with UV and visible light, we only observe the photocurrent effect without any gradual current change. Along with the Raman and PL characterizations, we demonstrate that the hybrid AZO/MoS 2 FETs show lightcontrolled doping effect via the photoisomerization of AZO molecules.

The drain current level can be controlled by the population ratio of trans-/cis-AZO molecules on the $\mathrm{MoS}_{2}$ surface. To trigger the molecular isomerization and adjust the trans/cis ratio we have used short-time UV light irradiation. The transfer curves reveal gradual current change and continuous threshold voltage shift as portrayed in Figure 4e and Figure S8. The inset in Figure 4e exhibits the time-resolved photoresponse of $\mathrm{AZO} / \mathrm{MoS}_{2} \mathrm{FET}$. After each UV irradiation cycle (time, $5 \mathrm{~s}$ ), the drain current decreases and hold at that current level. A total of four conductance 
states can be observed by 3 cycles of UV irradiation and the high conductance state can be recovered by long-time irradiation with visible light. Therefore, the hybrid AZO/MoS 2 FET can retain at multiple current levels by the remote light control. We also characterize the retention behavior of the hybrid $\mathrm{AZO} / \mathrm{MoS}_{2} \mathrm{FET}$. The metastable cis-AZO is thermodynamically unstable, thus it can be switched back to the trans-form by thermal energy. Figure $4 \mathrm{f}$ shows the transfer curves of the hybrid devices during the whole retention test. After UV irradiation, the drain current decreases and the threshold voltage shifts to the positive direction, demonstrating the low conductance state due to the $p$-doping of $\mathrm{MoS}_{2}$ FET by cis-AZO. Then the FET device is kept in the dark and we record the transfer curves of the FET after different storage time. The drain current and the threshold voltage exhibit very weak fluctuation. After the storage in the dark for more than 15 hours, the device is set back to the high conductance state by visible light irradiation. The inset in Figure $4 \mathrm{f}$ plots the drain current at $V_{\mathrm{g}}=60 \mathrm{~V}$ with the retention time and it reveals that the current of the hybrid cis-AZO/MoS 2 device is relatively stable ( $<1 \%$ variation) within 15 hours. In view of its high stability and the multiple current level which can be set by optical stimuli, the hybrid device is promising for the application in optical memory. Regarding the long switching time for photoisomerization of AZO molecules, future study on the improved switching speed will be beneficial for the practical application.

Resistor-diode transition in BP based lateral heterojunction. We have successfully demonstrated the light-controlled doping of $n$-type semiconductor $\mathrm{MoS}_{2}$ by the adsorption of AZO molecules. For the application of 2D semiconductors in electronic components, an ambipolar behavior, i.e. complementary $n$-type and $p$-type transistors with the same channel material, is preferable for developing CMOS like logic circuits, to achieve robust, low-power and low-cost 
smart electronics. In this context, it is essential to extend this doping methodology to other 2D semiconductors, especially for $p$-type or small bandgap semiconductors. Among the 2D semiconductors, few-layer BP FET is well-known for its ambipolar transport behavior with large tunability due to its small bandgap nature. ${ }^{44-46}$ The precise control of BP's charge carrier polarity and carrier density from the molecular approach is desirable for the electronic applications. The few-layer BP FET was fabricated on $\mathrm{BCB} / \mathrm{SiO}_{2} / \mathrm{Si}$ substrate with $\mathrm{Cr} / \mathrm{Au}(3 \mathrm{~nm} / 47 \mathrm{~nm})$ as top metal contact. The ambipolar transport behavior with dominant $p$-type transport is observed in pristine BP FET. The detailed characterization and discussion of pristine BP FET can be found in Supporting Information Figure S9.

We first demonstrate the light-controllable doping of BP FET by AZO molecules (device structure shown in Figure 5a, channel length/width is $2.2 \mu \mathrm{m} / 3.3 \mu \mathrm{m}$ ). Compared with BP FET without molecules, the adsorption of trans-AZO induces slight $p$-type doping feature (Figure S10). Figure $5 \mathrm{~b}$ portrays the transfer curves of the hybrid AZO/BP system for one cycle of light irradiation. As a result of the UV irradiation, the drain current increases at negative gate voltage region and it decreases at positive gate voltage region whereas the threshold voltages for both holes and electrons shift to the positive direction. Such response indicates the $p$-type doping of few-layer BP flake by cis-AZO molecules. The visible light triggers the back conversion of cis-AZO to transAZO. The drain current and the threshold voltage shift back to the previous condition. When the AZO is in its trans form, the field-effect mobility of BP is $32.75 \mathrm{~cm}^{2} \mathrm{~V}^{-1} \mathrm{~s}^{-1}$ for the holes and 17.40 $\mathrm{cm}^{2} \mathrm{~V}^{-1} \mathrm{~s}^{-1}$ for the electrons, whereas upon isomerization to cis form it amounts to 41.46 and 9.33 $\mathrm{cm}^{2} \mathrm{~V}^{-1} \mathrm{~s}^{-1}$ for the holes and electrons, respectively. The doping type of AZO molecules on BP is the same as that on $\mathrm{MoS}_{2}$. Such result indicates that dipolar interaction is the dominant doping mechanism. The shift of the threshold voltage upon AZO's isomerization induces a large current 
ratio $\left(I_{\text {cis }} / I_{\text {trans }}, \sim 10\right.$ to $\left.10^{2}\right)$ at $10 \mathrm{~V}<V_{\mathrm{g}}<25 \mathrm{~V}$, as displayed in Figure $5 \mathrm{c}$. At $20 \mathrm{~V}<V_{\mathrm{g}}<30 \mathrm{~V}$, BP FET with cis-AZO exhibits hole transport characteristics while BP FET with trans-AZO shows electron transport behavior. The output curves in Figure $5 \mathrm{~d}$ indicate the formation of good electrical contact for both cis- and trans-AZO/BP FET even under small drain voltage. Overall, light triggered isomerization of the AZO molecules makes it possible to achieve fine and local control of the carrier density and carrier type in BP, being a key step towards the construction of multifunctional devices.

By taking advantage of the capacity of adsorbed AZO to modulate reversibly the electronic properties of BP, in combination with standard micropatterning protocols, we have developed BP based lateral heterojunctions to achieve local control of the doping level in different regions of the channel. This was attained via the selective adsorption of AZO on patterned channel area and by exploiting the light tunable doping ability of AZO. Towards this end, a thin layer of PMMA was patterned by e-beam lithography to partially cover the channel of BP FET and the AZO molecules were adsorbed on the exposed channel area. The scheme of the device architecture is shown in Figure 6a. The junction consists of lateral heterojunction comprising a side-by-side BP and AZO/BP region. Thanks to the ambipolar transport behavior of BP FET, the electrostatic doping by gate can tune the polarity of BP transport. Under certain gate voltage, the pristine BP side switch to $n$-type transport, while the AZO/BP side remains the $p$-type transport due to the $p$-doping effect of $c i s-\mathrm{AZO}$. This contributes to the formation of $p$ - $n$ junction in the lateral heterojunction structure. Figure $6 \mathrm{~b}$ shows the gate-dependent output curves of BP based lateral heterojunction with selective doping by cis-AZO molecules. The inset shows the logarithmic plot of the output curves. We observe the obvious rectifying behavior under $V_{\mathrm{g}}$ from $10 \mathrm{~V}$ to $30 \mathrm{~V}$. The rectification ratio, defined as the ratio of the forward/reverse current, increases as $V_{\mathrm{g}}$ decreases. At $V_{\mathrm{g}}=10 \mathrm{~V}$, a rectification 
ratio of $\sim 600$ is obtained at $V_{\mathrm{d}}= \pm 0.3 \mathrm{~V}$. The minimum ideal factor is 1.82 with $V_{\mathrm{g}}=10 \mathrm{~V}$. The large ideal factor is related to the Schottky barrier at $\mathrm{BP} / \mathrm{Cr}$ contract and the $\mathrm{BP}$ crystal defects or surface oxidation. ${ }^{47,48}$. The current-rectifying effect can also be demonstrated from the transfer curves at positive and negative drain voltage as shown in Figure $6 \mathrm{c}$. When $V_{\mathrm{g}}$ is in the range of 10 $\mathrm{V}$ to $30 \mathrm{~V}$, the drain current shows orders of magnitude difference under positive and negative drain voltage. At negative $V_{\mathrm{d}}$, the drain current presents a plateau region with minimum current level, while at positive $V_{\mathrm{d}}$, the drain current shows normal ambipolar transport behavior. Under other gate voltage bias, the drain currents display little difference at positive and negative drain voltage. From the band alignment of BP based lateral heterojunction, we can understand the gatedependent current-rectifying behavior. The Fermi level of BP can be tuned by the back-gate bias and the molecular doping. When the gate voltage is negative, the FET acts as a $p-p$ junction, which shows negligible rectification effect. When the gate voltage exceeds $30 \mathrm{~V}$, the device behaves as $n$ - $n$ junction and the low drain current is limited by the $\mathrm{BP} /$ metal contact and the intrinsic charge trap. Therefore, in the above gate bias condition, the transfer curves are almost overlapped under positive and negative drain voltage. When the gate voltage is between $10 \mathrm{~V}$ and $30 \mathrm{~V}$, the BP side transfer to $n$-type transport and the $c i s$-AZO/BP side remains $p$-type transport, which results in the $p-n$ junction (inset of Figure 6c).

The cis-AZO can convert back to the trans form by the visible light irradiation and the transport behavior of the patterned AZO/BP FET with trans-AZO are shown in Supporting Information Figure S11. The output curves reveal that the current-rectifying effect is quite weak and even disappearing. Figure $6 \mathrm{~d}$ summarizes the rectification ratio of $\mathrm{BP}$ based lateral heterojunction under different molecules state and gate voltage. It reveals that with cis-AZO doping, the hybrid lateral heterojunction device exhibits gate-dependent $p-p, p-n, n-n$ junction transition and dramatic 
change of the rectification ratio, while with trans-AZO, the current rectification effect is rather weak. Therefore, the functional devices based on the patterned hybrid AZO/BP FET can switch between diodes and resistors under light control. Such a patterning-based approach was also applied to $\mathrm{AZO} / \mathrm{MoS}_{2}$ FETs. The output curves displayed in Supporting Information Figure S12 reveal that the current-rectifying behavior has very weak dependence on the state of the molecules due to the unipolar transport behavior of $\mathrm{MoS}_{2}$. All these results demonstrate that the ambipolar transport behavior of $\mathrm{BP}$ and the $p$-type doping of cis-AZO molecules synergically contribute to the light-controlled resistors-diode transition on the patterned AZO/BP functional devices. Compared with the previously reported photoswitchable diode based on metal-semiconductor Schottky junction, ${ }^{20}$ the patterned AZO/BP $p$ - $n$ diode adopts the commonly used FET architecture and shows the ability of spatially tunable doping, which is ideal for the practical fabrication of functional devices.

\section{Conclusion}

In this work, we have demonstrated a universal approach to optically switch $2 \mathrm{D}$ electronics by fabricating hybrid vdWHs comprising 2D semiconductors coated with photochromic adlayer, supported on trap-free polymer dielectric. The improved 2D/dielectric interface excludes the unfavorable influence from substrate, thereby minimizing the effect of PPC, and optimizes the device performance, being a prerequisite to the realization of switchable devices. The lightinduced geometry change of AZO molecules leads to the redistribution of dipole moment on $2 \mathrm{D}$ surface and effectively manipulates the carrier concentration in 2D semiconductors with light as remote control. Both the spectroscopic characterization and the electronic transport measurement 
provided unambiguous evidence for the high efficiency in tuning reversibly the doping of $2 \mathrm{D}$ semiconductors by light-control over the isomerization of the photochromic molecules. Noteworthy, the achieved modulation over the carrier concentration along with the preserved high FET performance are essential for functional electronic devices, including optical memory and $p$ $n$ diodes. The electronic devices based on the hybrid vertical vdWHs displayed multiple current levels with excellent stability and the switchable resistor-diode transition with spatially tunable doping.

Interface engineering is of vital importance for the electronics of 2D semiconductors, due to their high surface-to-volume ratio. The fine tuning of the interfaces of the 2D materials with both the bottom dielectric substrate surface and the top molecular adlayer enabled us to develop efficient light-responsive vdWHs. Our universal approach offers a guideline for enriching 2D electronics with additional functionalities imparted by ad-hoc molecular switches. The future study on the formation of ordered molecular structures can maximize the collective molecular dipole moment change and extend the carrier modulation range. Such a strategy can be exploited with molecules responding to magnetic fields and/or electrochemical stimuli, thus making it possible to realize the chemical tailoring of hybrid vdWHs with electronic, magnetic and electrochemical functions. It is also worth noting that our method can be applicable for the fabrication of large-scale device arrays and systems. The molecular engineering of $2 \mathrm{D}$ semiconductors in combination with unconventional device architectures will lay the foundation for the next generation of highperformance $2 \mathrm{D}$ electronics based on functional and multiresponsive devices.

\section{Experimental Methods}


Materials. Bulk $\mathrm{MoS}_{2}$ and BP crystals were purchased from commercial vendors (SPI Supplies and HQ graphene). The 4-(decyloxy) azobenzene molecules (Product No. S931950) were purchased from Sigma-Aldrich. The BCB polymer, divinyltetramethyldisiloxanebis(benzocyclobutene), was purchased from Dow Chemical (CYCLOTENE ${ }^{\mathrm{TM}}$ ) and diluted with mesitylene solution.

Optical spectroscopy characterization. UV-Vis absorption spectra of the 4-(decyloxy) azobenzene molecules were characterized with a JASCO V-670 spectrophotometer and the molecules were drop-casted onto quartz substrate. The molecular switching was triggered with fiber coupled LEDs (wavelength $365 \mathrm{~nm}$ and $455 \mathrm{~nm}$, ThorLabs). The LEDs power density was set to $1.5 \mathrm{~mW} / \mathrm{cm}^{2}$ for $365 \mathrm{~nm}$ and $2.5 \mathrm{~mW} / \mathrm{cm}^{2}$ for $455 \mathrm{~nm}$ by the LED driver. The Raman and PL spectroscopy of monolayer $\mathrm{MoS}_{2}$ with the adsorption of AZO molecules were acquired with a Renishaw InVia spectrometer equipped with a $532 \mathrm{~nm}$ laser. Monolayer $\mathrm{MoS}_{2}$ flakes were mechanically exfoliated from $\mathrm{MoS}_{2}$ crystals onto $\mathrm{SiO}_{2} / \mathrm{Si}$ substrate. The AZO molecules are dissolved in ethanol solutions with the concentration of $0.2 \mathrm{mg} / \mathrm{mL}$. In the deposition process, the molecules are spin-coated onto the surface of 2D semiconductor flakes with $1000 \mathrm{rpm}$ speed. A mild annealing at $50{ }^{\circ} \mathrm{C}$ for one hour is conducted to evaporate the solvent. The excitation power of the laser was kept below $1 \mathrm{~mW}$ to avoid the local heating and damaging effect on the samples. The Si peak at $520.5 \mathrm{~cm}^{-1}$ was used for normalization.

AFM. The AFM characterization was carried out by means of a Veeco Multimode V microscope equipped with a Nanoscope $\mathrm{V}$ controller operating in intermittent contact mode. Both height and phase images have been recorded by using tips for a force constant $42 \mathrm{~N} / \mathrm{m}$. 
Device fabrication and characterization. The $\mathrm{BCB}$ polymer was spin-coated onto the thermally oxidized silicon substrate $\left(t_{\mathrm{ox}}=270 \mathrm{~nm}\right)$ and crosslinked to the final gate dielectric by postannealing at $290{ }^{\circ} \mathrm{C}(10 \mathrm{~min}$ in a nitrogen-filled glovebox). The thickness of the BCB polymer (60 nm) was determined by an Alpha-Step IQ Surface Profiler from KLA Tencor. The dielectric constant of $\mathrm{BCB}$ is $\sim 2.65$ and the capacitance of the $\mathrm{BCB} / \mathrm{SiO}_{2}$ bilayer dielectric is $9.64 \mathrm{nF} / \mathrm{cm}^{2}$. 2D semiconductors, including $\mathrm{MoS}_{2}$ and BP, are mechanically exfoliated by the scotch tape-based method and transferred to the $\mathrm{BCB} / \mathrm{SiO}_{2}$ bilayer dielectric substrate and $\mathrm{SiO}_{2}$ substrate. Bake-gated FETs were fabricated by e-beam lithography and the metal electrodes were thermally evaporated onto the patterned substrate. The lift-off process was carried out in warm acetone $\left(50^{\circ} \mathrm{C}\right)$ and the as-fabricated devices were annealed at $150{ }^{\circ} \mathrm{C}$ for $12 \mathrm{~h}$ in a high-vacuum chamber to desorb atmospheric adsorbates. In order to fabricate the BP homojunction, a thin layer of PMMA was spin-coated onto the BP FET and patterned to partially cover the BP channel by e-beam lithography. All the devices were kept in a nitrogen-filled glovebox after annealing and electrically characterized in a probe station connected to a Keithley 2636. For the light-induced switching, a Polychrome V monochromator (Till Photonics) was used as UV (365 nm, $\left.9.69 \mathrm{~mW} / \mathrm{cm}^{2}\right)$ and visible light source $\left(455 \mathrm{~nm}, 23.88 \mathrm{~mW} / \mathrm{cm}^{2}\right)$ to irradiate vertically onto the FET.

The carrier mobility $\mu$ was determined by the following equation:

$$
\mu=\frac{d I_{d}}{d V_{g}} \times \frac{L}{W C_{i} V_{d}}
$$

where $L$ and $W$ are the channel length and width, and $C_{\mathrm{i}}$ is the capacitance per unit area.

The pristine carrier density $\mathrm{n}_{2 D}$ in $2 \mathrm{DMs}$ can be extracted from the formula: ${ }^{49}$ 


$$
\mathrm{n}_{2 D}=\frac{\sigma_{2 D}}{q \mu}=\frac{I_{d} L}{V_{d} W q \mu}
$$

where $\sigma_{2 D}$ is the conductivity of $2 \mathrm{D}$ sheet, and $q$ is the elementary charge.

The interface trap density $\left(D_{\text {it }}\right)$ can be calculated based on the following equation, ${ }^{50}$

$$
\mathrm{SS}=\left(\frac{d\left(\log _{10} I_{D}\right)}{d V_{g}}\right)^{-1} \approx \ln 10 \frac{k T}{q}\left(1+\frac{q D_{i t}}{C_{i}}\right)
$$

where $S S$ is the subthreshold swing, $k$ is the Boltzmann constant, $T$ is the temperature, $q$ is the elementary charge, and $C_{\mathrm{i}}$ is the capacitance of the dielectric per unit area. The SS value of $\mathrm{MoS}_{2}$ transistors is $3.55 \mathrm{~V} / \mathrm{dec}$ on $\mathrm{SiO}_{2}$ and $2.89 \mathrm{~V} / \mathrm{dec}$ on $\mathrm{BCB}$ and the capacitance of $\mathrm{SiO}_{2}$ and $\mathrm{BCB} / \mathrm{SiO}_{2}$ is $12.79 \mathrm{nF} / \mathrm{cm}^{2}$ and $9.64 \mathrm{nF} / \mathrm{cm}^{2}$, respectively. Thus, the estimated $D_{\mathrm{it}}$ value is $4.69 \times$ $10^{12} \mathrm{~cm}^{-2} \mathrm{eV}^{-1}$ and $2.86 \times 10^{12} \mathrm{~cm}^{-2} \mathrm{eV}^{-1}$ at the $\mathrm{MoS}_{2} /$ dielectric interface without and with $\mathrm{BCB}$, respectively.

The isomerization-induced charge density was calculated by:

$$
\Delta \mathrm{n}=C_{i} \frac{\Delta V}{q}
$$

where $\Delta V$ is the difference in the threshold voltage $\left(V_{\text {th }}\right)$ before and after isomerization.

We calculate the ideal factor of the $p$ - $n$ diode based on the following Shockley diode equation: ${ }^{.1}$

$$
I=I_{S}\left(e^{\frac{q V_{d}}{n k T}}-1\right)
$$

where $I$ is the diode current, $I_{\mathrm{s}}$ is the reverse bias saturation current, $V_{\mathrm{d}}$ is the voltage across the diode, $n$ is the ideal factor, and $k T / q$ is the thermal voltage.

\section{ASSOCIATED CONTENT}


The authors declare no competing interests.

\section{Supporting Information.}

The Supporting Information is available free of charge on the ACS Publications website at DOI: $\operatorname{XXXXX}$.

Figures S1-S12 and references (refs 1-3). The isomerization of AZO molecules, photoresponse characterization of pristine $\mathrm{MoS}_{2}$ FET and hybrid $\mathrm{MoS}_{2}$ FET with molecules, and the electrical characterization of pristine BP FET, BP and $\mathrm{MoS}_{2}$ lateral heterojunction. (PDF)

\section{AUTHOR INFORMATION}

Corresponding Author

*E-mail: samori@unistra.fr

ORCID

Paolo Samorì: 0000-0001-6256-8281

Author Contributions

Y.Z., S.B. and P.S. conceived the experiment. Y.Z. performed the AFM study, spectroscopic characterization and carried out the device fabrication and characterization. Y.Z. and P.S. cowrote the paper. All authors discussed the results and contributed to the interpretation of data as well as to editing the manuscript.

\section{ACKNOWLEDGMENT}

Device fabrication was carried out in part at the nanotechnology facility eFab (IPCMS, Strasbourg). We acknowledge funding from the European Commission through the Graphene 
Flagship Core 2 project (GA-785219), the Marie Sklodowska-Curie projects ITN project iSwitch (GA-642196), the Marie-Curie IEF MULTI2DSWITCH (GA-700802) and the Marie-Curie IEF STELLAR (GA-795615), the M-ERA.NET project MODIGLIANI, the Agence Nationale de la Recherche through the Labex projects CSC (ANR-10-LABX-0026 CSC) and NIE (ANR-11LABX-0058 NIE) within the Investissement d'Avenir program (ANR-10-120 IDEX-0002-02), and the International Center for Frontier Research in Chemistry (icFRC).

\section{REFERENCES}

1. Geim, A. K.; Grigorieva, I. V. Van der Waals Heterostructures. Nature 2013, 499, 419-425. 2. Liu, Y.; Weiss, N. O.; Duan, X.; Cheng, H.-C.; Huang, Y.; Duan, X. Van der Waals Heterostructures and Devices. Nat. Rev. Mater. 2016, 1, 16042.

3. Butler, S. Z.; Hollen, S. M.; Cao, L.; Cui, Y.; Gupta, J. A.; Gutierrez, H. R.; Heinz, T. F.; Hong, S. S.; Huang, J.; Ismach, A. F.; Johnston-Halperin, E.; Kuno, M.; Plashnitsa, V. V.; Robinson, R. D.; Ruoff, R. S.; Salahuddin, S.; Shan, J.; Shi, L.; Spencer, M. G.; Terrones, M., et al. Progress, Challenges, and Opportunities in Two-Dimensional Materials Beyond Graphene. ACS Nano 2013, 7, 2898-2926.

4. Novoselov, K. S.; Mishchenko, A.; Carvalho, A.; Castro Neto, A. H. 2D Materials and van der Waals Heterostructures. Science 2016, 353, aac9439.

5. Jariwala, D.; Sangwan, V. K.; Lauhon, L. J.; Marks, T. J.; Hersam, M. C. Emerging Device Applications for Semiconducting Two-Dimensional Transition Metal Dichalcogenides. ACS Nano 2014, $8,1102-1120$.

6. Jariwala, D.; Marks, T. J.; Hersam, M. C. Mixed-Dimensional van der Waals Heterostructures. Nat. Mater. 2016, 16, 170.

7. Gobbi, M.; Orgiu, E.; Samorì, P. When 2D Materials Meet Molecules: Opportunities and Challenges of Hybrid Organic/Inorganic van der Waals Heterostructures. Adv. Mater. 2018, 30, 1706103 .

8. Szaciłowski, K. Digital Information Processing in Molecular Systems. Chem. Rev. 2008, 108, 3481-3548.

9. Kathan, M.; Hecht, S. Photoswitchable Molecules As Key Ingredients to Drive Systems away from the Global Thermodynamic Minimum. Chem. Soc. Rev. 2017, 46, 5536-5550.

10. Zhang, X.; Hou, L.; Samorì, P. Coupling Carbon Nanomaterials with Photochromic Molecules for the Generation of Optically Responsive Materials. Nat. Commun. 2016, 7, 11118.

11. Chamlagain, B.; Li, Q.; Ghimire, N. J.; Chuang, H.-J.; Perera, M. M.; Tu, H.; Xu, Y.; Pan, M.; Xaio, D.; Yan, J.; Mandrus, D.; Zhou, Z. Mobility Improvement and Temperature Dependence in $\mathrm{MoSe}_{2}$ Field-Effect Transistors on Parylene-C Substrate. ACS Nano 2014, 8, 5079-5088.

12. Liu, B.; Zhao, W.; Ding, Z.; Verzhbitskiy, I.; Li, L.; Lu, J.; Chen, J.; Eda, G.; Loh Kian, P. Engineering Bandgaps of Monolayer $\mathrm{MoS}_{2}$ and $\mathrm{WS}_{2}$ on Fluoropolymer Substrates by Electrostatically Tuned Many-Body Effects. Adv. Mater. 2016, 28, 6457-6464.

13. Liu, X.; Hersam, M. C. Interface Characterization and Control of 2D Materials and 
Heterostructures. Adv. Mater. 2018, 30, 1801586.

14. Wood, J. D.; Wells, S. A.; Jariwala, D.; Chen, K.-S.; Cho, E.; Sangwan, V. K.; Liu, X.; Lauhon, L. J.; Marks, T. J.; Hersam, M. C. Effective Passivation of Exfoliated Black Phosphorus Transistors against Ambient Degradation. Nano Lett. 2014, 14, 6964-6970.

15. Choi, M. S.; Qu, D.; Lee, D.; Liu, X.; Watanabe, K.; Taniguchi, T.; Yoo, W. J. Lateral MoS p-n Junction Formed by Chemical Doping for Use in High-Performance Optoelectronics. ACS Nano 2014, 8, 9332-9340.

16. Pala, R. A.; Shimizu, K. T.; Melosh, N. A.; Brongersma, M. L. A Nonvolatile Plasmonic Switch Employing Photochromic Molecules. Nano Lett. 2008, 8, 1506-1510.

17. Kim, M.; Safron, N. S.; Huang, C.; Arnold, M. S.; Gopalan, P. Light-Driven Reversible Modulation of Doping in Graphene. Nano Lett. 2012, 12, 182-187.

18. Margapoti, E.; Strobel, P.; Asmar, M. M.; Seifert, M.; Li, J.; Sachsenhauser, M.; Ceylan, Ö.; Palma, C.-A.; Barth, J. V.; Garrido, J. A.; Cattani-Scholz, A.; Ulloa, S. E.; Finley, J. J. Emergence of Photoswitchable States in a Graphene-Azobenzene-Au Platform. Nano Lett. 2014, $14,6823-6827$.

19. Döbbelin, M.; Ciesielski, A.; Haar, S.; Osella, S.; Bruna, M.; Minoia, A.; Grisanti, L.; Mosciatti, T.; Richard, F.; Prasetyanto, E. A.; De Cola, L.; Palermo, V.; Mazzaro, R.; Morandi, V.; Lazzaroni, R.; Ferrari, A. C.; Beljonne, D.; Samorì, P. Light-Enhanced Liquid-Phase Exfoliation and Current Photoswitching in Graphene-Azobenzene Composites. Nat. Commun. 2016, 7, 11090. 20. Margapoti, E.; Li, J.; Ceylan, Ö.; Seifert, M.; Nisic, F.; Anh, T. L.; Meggendorfer, F.; Dragonetti, C.; Palma, C.-A.; Barth, J. V.; Finley, J. J. A 2D Semiconductor-Self-Assembled Monolayer Photoswitchable Diode. Adv. Mater. 2015, 27, 1426-1431.

21. Gobbi, M.; Bonacchi, S.; Lian, J. X.; Vercouter, A.; Bertolazzi, S.; Zyska, B.; Timpel, M.; Tatti, R.; Olivier, Y.; Hecht, S.; Nardi, M. V.; Beljonne, D.; Orgiu, E.; Samorì, P. Collective Molecular Switching in Hybrid Superlattices for Light-Modulated Two-Dimensional Electronics. Nat. Commun. 2018, 9, 2661.

22. Wu, Y.-C.; Liu, C.-H.; Chen, S.-Y.; Shih, F.-Y.; Ho, P.-H.; Chen, C.-W.; Liang, C.-T.; Wang, W.-H. Extrinsic Origin of Persistent Photoconductivity in Monolayer $\mathrm{MoS}_{2}$ Field Effect Transistors. Sci. Rep. 2015, 5, 11472 .

23. Lopez-Sanchez, O.; Lembke, D.; Kayci, M.; Radenovic, A.; Kis, A. Ultrasensitive Photodetectors Based on Monolayer MoS. Nat. Nanotechnol. 2013, 8, 497-501.

24. Klajn, R. Spiropyran-Based Dynamic Materials. Chem. Soc. Rev. 2014, 43, 148-184.

25. Min, M.; Seo, S.; Lee, S. M.; Lee, H. Voltage-Controlled Nonvolatile Molecular Memory of an Azobenzene Monolayer through Solution-Processed Reduced Graphene Oxide Contacts. Adv. Mater. 2013, 25, 7045-7050.

26. Wang, J.; Yu, H.; Zhou, X.; Liu, X.; Zhang, R.; Lu, Z.; Zheng, J.; Gu, L.; Liu, K.; Wang, D.; Jiao, L. Probing the Crystallographic Orientation of Two-Dimensional Atomic Crystals with Supramolecular Self-Assembly. Nat. Commun. 2017, 8, 377.

27. Osella, S.; Minoia, A.; Beljonne, D. Combined Molecular Dynamics and Density Functional Theory Study of Azobenzene-Graphene Interfaces. J. Phys. Chem. C 2016, 120, 66516658.

28. Fihey, A.; Perrier, A.; Browne, W. R.; Jacquemin, D. Multiphotochromic Molecular Systems. Chem. Soc. Rev. 2015, 44, 3719-3759.

29. Ferrari, A. C.; Basko, D. M. Raman Spectroscopy As a Versatile Tool for Studying the Properties of Graphene. Nat. Nanotechnol. 2013, 8, 235-246.

30. Wei, S.; Miao-Ling, L.; Qing-Hai, T.; Xiao-Fen, Q.; Jun, Z.; Ping-Heng, T. Raman and 
Photoluminescence Spectra of Two-Dimensional Nanocrystallites of Monolayer $\mathrm{WS}_{2}$ and $\mathrm{WSe}_{2}$. 2D Mater. 2016, 3, 025016.

31. Zeng, H.; Cui, X. An Optical Spectroscopic Study on Two-Dimensional Group-VI Transition Metal Dichalcogenides. Chem. Soc. Rev. 2015, 44, 2629-2642.

32. Li, H.; Zhang, Q.; Yap, C. C. R.; Tay, B. K.; Edwin, T. H. T.; Olivier, A.; Baillargeat, D. From Bulk to Monolayer $\mathrm{MoS}_{2}$ : Evolution of Raman Scattering. Adv. Funct. Mater. 2012, 22, 13851390.

33. Chakraborty, B.; Bera, A.; Muthu, D. V. S.; Bhowmick, S.; Waghmare, U. V.; Sood, A. K. Symmetry-Dependent Phonon Renormalization in Monolayer $\mathrm{MoS}_{2}$ Transistor. Phys. Rev. B 2012, $85,161403$.

34. Rice, C.; Young, R. J.; Zan, R.; Bangert, U.; Wolverson, D.; Georgiou, T.; Jalil, R.; Novoselov, K. S. Raman-Scattering Measurements and First-Principles Calculations of StrainInduced Phonon Shifts in Monolayer MoS. Phys. Rev. B 2013, 87, 081307.

35. Mak, K. F.; He, K.; Lee, C.; Lee, G. H.; Hone, J.; Heinz, T. F.; Shan, J. Tightly Bound Trions in Monolayer $\mathrm{MoS}_{2}$. Nat. Mater. 2012, 12, 207.

36. Zhang, W.; Huang, J.-K.; Chen, C.-H.; Chang, Y.-H.; Cheng, Y.-J.; Li, L.-J. High-Gain Phototransistors Based on a CVD $\mathrm{MoS}_{2}$ Monolayer. Adv. Mater. 2013, 25, 3456-3461.

37. Chua, L.-L.; Zaumseil, J.; Chang, J.-F.; Ou, E. C. W.; Ho, P. K. H.; Sirringhaus, H.; Friend, R. H. General Observation of n-Type Field-Effect Behaviour in Organic Semiconductors. Nature 2005, 434, 194.

38. Liu, Y.; Guo, J.; Zhu, E.; Liao, L.; Lee, S.-J.; Ding, M.; Shakir, I.; Gambin, V.; Huang, Y.; Duan, X. Approaching the Schottky-Mott Limit in van der Waals Metal-Semiconductor Junctions. Nature 2018, 557, 696-700.

39. Cui, X.; Lee, G.-H.; Kim, Y. D.; Arefe, G.; Huang, P. Y.; Lee, C.-H.; Chenet, D. A.; Zhang, X.; Wang, L.; Ye, F.; Pizzocchero, F.; Jessen, B. S.; Watanabe, K.; Taniguchi, T.; Muller, D. A.; Low, T.; Kim, P.; Hone, J. Multi-Terminal Transport Measurements of $\mathrm{MoS}_{2}$ Using a van der Waals Heterostructure Device Platform. Nat. Nanotechnol. 2015, 10, 534-540.

40. Kang, Y.; Han, S. An Origin of Unintentional Doping in Transition Metal Dichalcogenides: the Role of Hydrogen Impurities. Nanoscale 2017, 9, 4265-4271.

41. Scheuschner, N.; Ochedowski, O.; Kaulitz, A.-M.; Gillen, R.; Schleberger, M.; Maultzsch, J. Photoluminescence of Freestanding Single- and Few-Layer MoS . Phys. Rev. B 2014, 89, 125406. 42. Zhao, Y.; Xu, K.; Pan, F.; Zhou, C.; Zhou, F.; Chai, Y. Doping, Contact and Interface Engineering of Two-Dimensional Layered Transition Metal Dichalcogenides Transistors. Adv. Funct. Mater. 2017, 27, 1603484.

43. Li, S.-L.; Tsukagoshi, K.; Orgiu, E.; Samorì, P. Charge Transport and Mobility Engineering in Two-Dimensional Transition Metal Chalcogenide Semiconductors. Chem. Soc. Rev. 2016, 45, 118-151.

44. Li, L. K.; Yu, Y. J.; Ye, G. J.; Ge, Q. Q.; Ou, X. D.; Wu, H.; Feng, D. L.; Chen, X. H.; Zhang, Y. B. Black Phosphorus Field-Effect Transistors. Nat. Nanotechnol. 2014, 9, 372-377.

45. Perello, D. J.; Chae, S. H.; Song, S.; Lee, Y. H. High-Performance n-Type Black Phosphorus Transistors with Type Control via Thickness and Contact-Metal Engineering. Nat. Commun. 2015, 6, 7809 .

46. Du, Y.; Liu, H.; Deng, Y.; Ye, P. D. Device Perspective for Black Phosphorus Field-Effect Transistors: Contact Resistance, Ambipolar Behavior, and Scaling. ACS Nano 2014, 8, 1003510042.

47. Deng, Y.; Luo, Z.; Conrad, N. J.; Liu, H.; Gong, Y.; Najmaei, S.; Ajayan, P. M.; Lou, J.; 
$\mathrm{Xu}, \mathrm{X}$;; Ye, P. D. Black Phosphorus-Monolayer $\mathrm{MoS}_{2}$ van der Waals Heterojunction p-n Diode. ACS Nano 2014, 8, 8292-8299.

48. Buscema, M.; Groenendijk, D. J.; Steele, G. A.; van der Zant, H. S. J.; Castellanos-Gomez, A. Photovoltaic Effect in Few-Layer Black Phosphorus PN Junctions Defined by Local Electrostatic Gating. Nat. Commun. 2014, 5, 4651.

49. Fang, H.; Tosun, M.; Seol, G.; Chang, T. C.; Takei, K.; Guo, J.; Javey, A. Degenerate nDoping of Few-Layer Transition Metal Dichalcogenides by Potassium. Nano Lett. 2013, 13, 19911995.

50. Klauk, H. Organic Thin-Film Transistors. Chem. Soc. Rev. 2010, 39, 2643-2666.

51. Shockley, W. The Theory of p-n Junctions in Semiconductors and p-n junction Transistors. Bell Syst. Tech.J. 1949, 28, 435-489. 
(a)

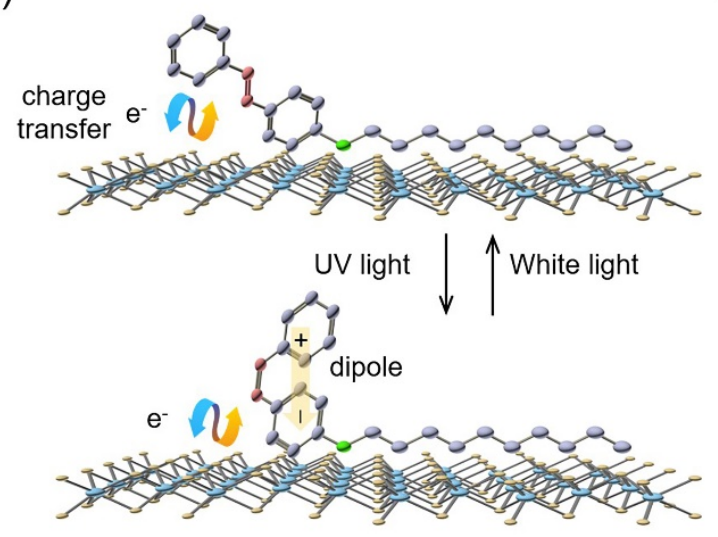

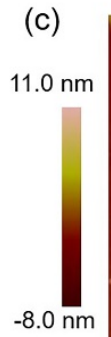
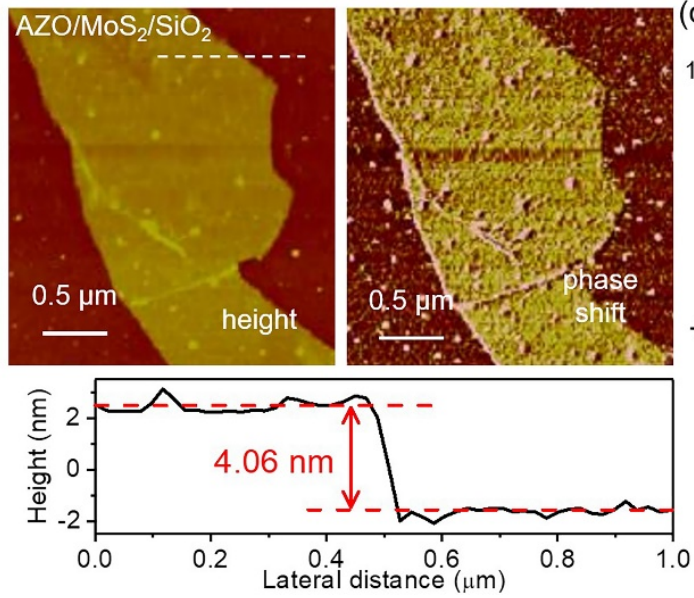
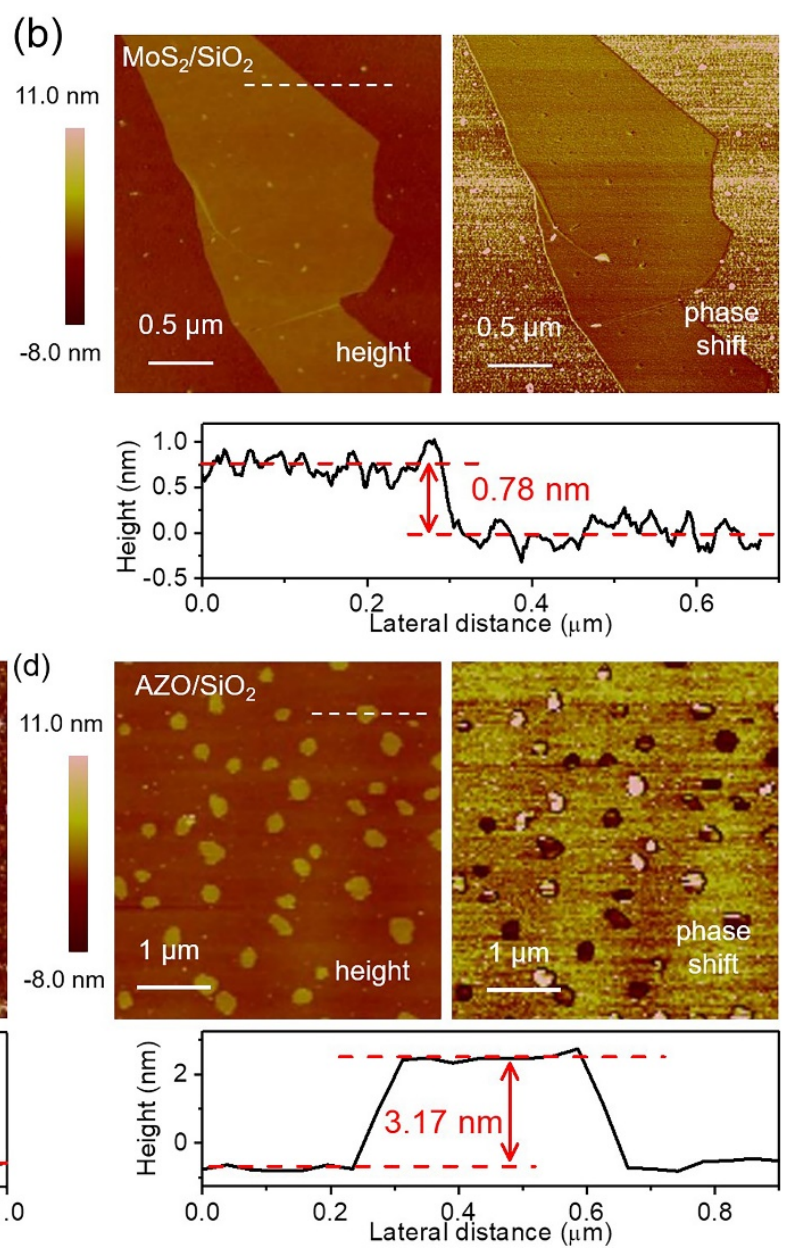

Figure 1. Morphological characterization of the $\mathrm{AZO} / \mathrm{MoS}_{2}$ hybrid system. (a) Schematic of the hybrid system based on photochromic molecules (4-(decyloxy) azobenzene) and 2D semiconductors $\left(\mathrm{MoS}_{2}\right)$, and model of the azobenzene's reversible photoisomerization. Intermittent contact mode AFM images of (b) monolayer $\mathrm{MoS}_{2}$, (c) the $\mathrm{AZO} / \mathrm{MoS}_{2}$ hybrid system, and (d) $\mathrm{AZO}$ molecules on $\mathrm{SiO}_{2} / \mathrm{Si}$ substrate including height image (left), phase image (right), and the recorded line profile. 
(a)

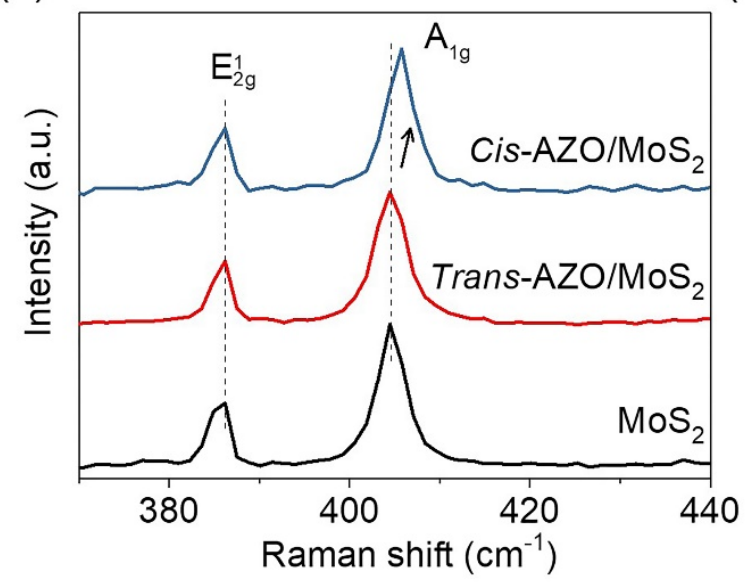

(b)

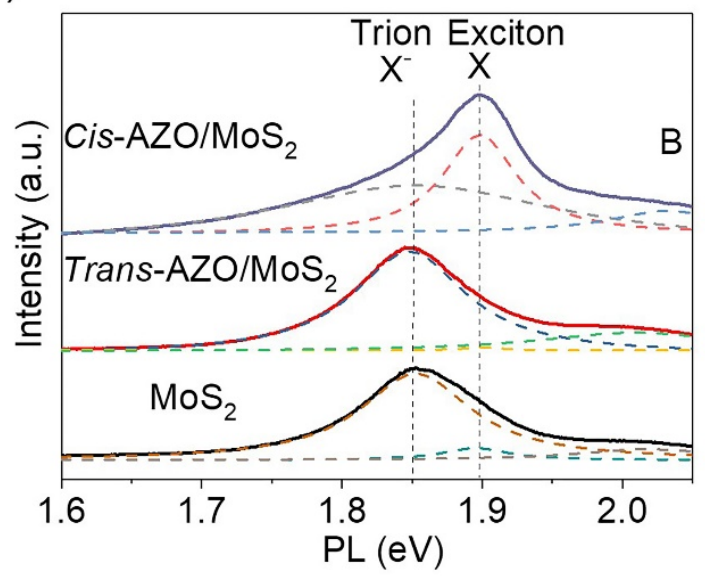

Figure 2. Spectroscopy characterization of the $\mathrm{AZO} / \mathrm{MoS}_{2}$ hybrid system. (a) Raman spectra of the pristine $\mathrm{MoS}_{2}$ and $\mathrm{AZO} / \mathrm{MoS}_{2}$ hybrid system, with cis-AZO and trans-AZO, respectively. (b) PL spectra of the pristine $\mathrm{MoS}_{2}$ and $\mathrm{AZO} / \mathrm{MoS}_{2}$ hybrid system, with cis-AZO and trans-AZO, respectively. Dashed line shows the fitted spectra of A exciton with trions $\mathrm{X}^{-}$and neutral excitons $\mathrm{X}$, and $\mathrm{B}$ exciton. 
(a)

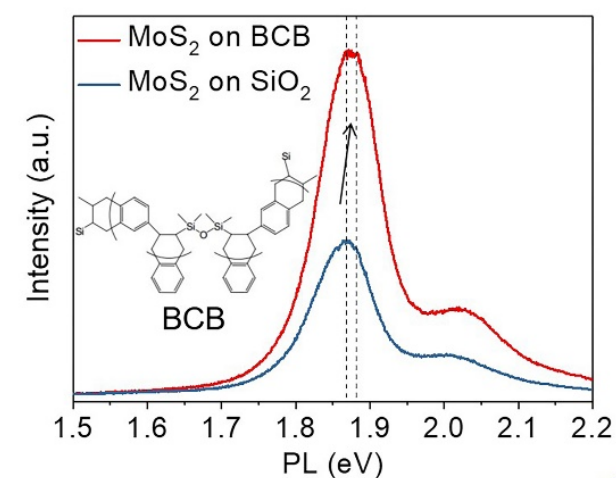

(c)
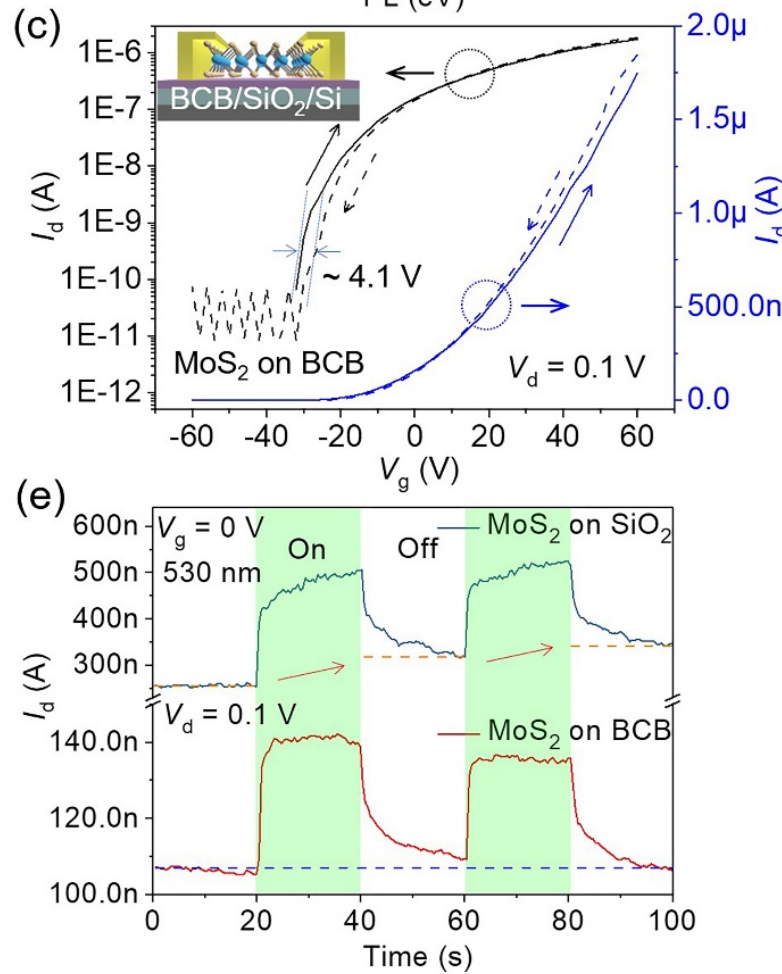

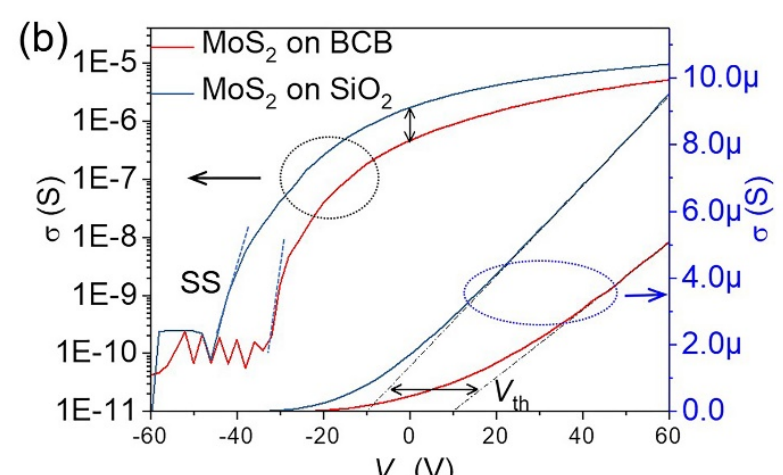

(d)

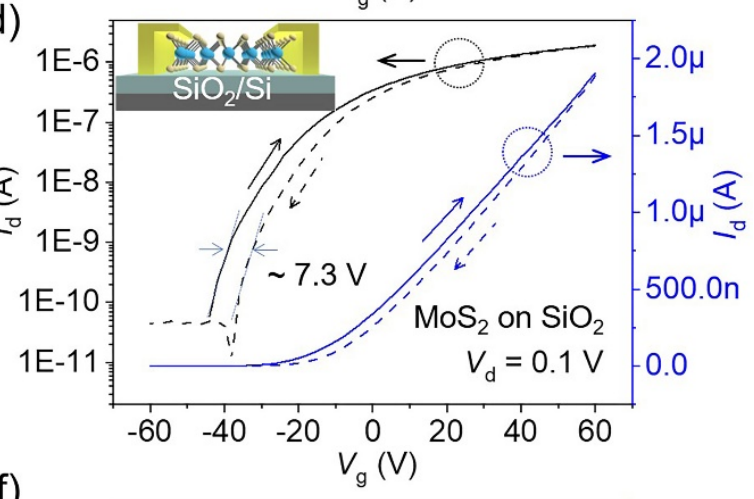

(f)

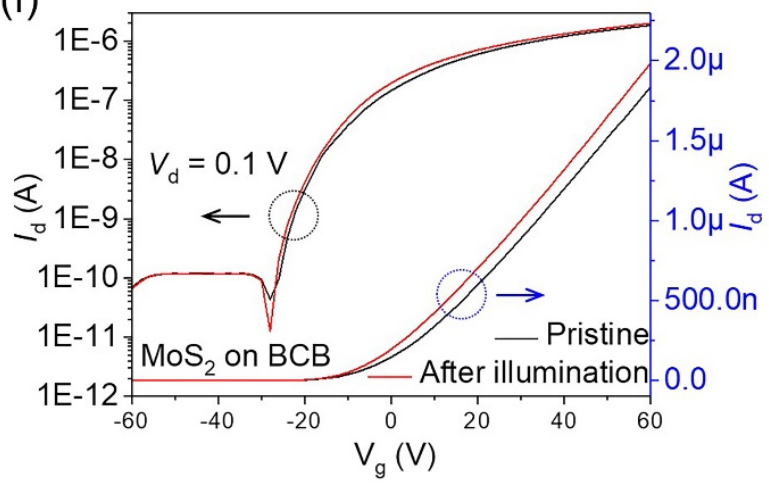

Figure 3. Comparison of $\mathrm{MoS}_{2} \mathrm{FET}$ on $\mathrm{BCB}$ and $\mathrm{SiO}_{2}$ substrate. (a) $\mathrm{PL}$ spectra of the monolayer $\mathrm{MoS}_{2}$ on $\mathrm{BCB}$ and $\mathrm{SiO}_{2}$ substrate. (b) Transfer curves of monolayer $\mathrm{MoS}_{2}$ FET on $\mathrm{BCB}$ and $\mathrm{SiO}_{2}$ substrate. Hysteresis test: transfer curves of monolayer $\mathrm{MoS}_{2}$ for one gate sweeping cycle on (c) $\mathrm{BCB} / \mathrm{SiO}_{2} / \mathrm{Si}$ substrate, and (d) $\mathrm{SiO}_{2} / \mathrm{Si}$ substrate. Inset shows the schematic of the device structure. The hysteresis decreases from $7.3 \mathrm{~V}$ on $\mathrm{SiO}_{2}$ substrate to $4.1 \mathrm{~V}$ on $\mathrm{BCB}$ substrate. (e) Time-resolved photoresponse of $\mathrm{MoS}_{2}$ FET on $\mathrm{BCB}$ and $\mathrm{SiO}_{2}$ substrate under $530 \mathrm{~nm}$ light illumination. (f) Transfer curves of $\mathrm{MoS}_{2}$ FET on BCB substrate before and after light illumination. 
(a)

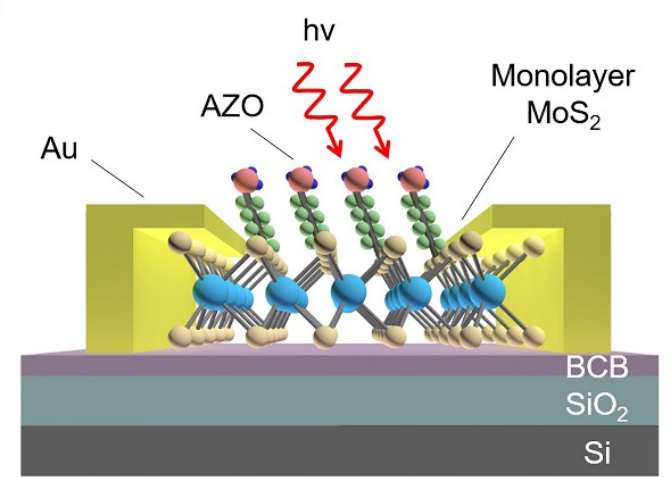

(c)

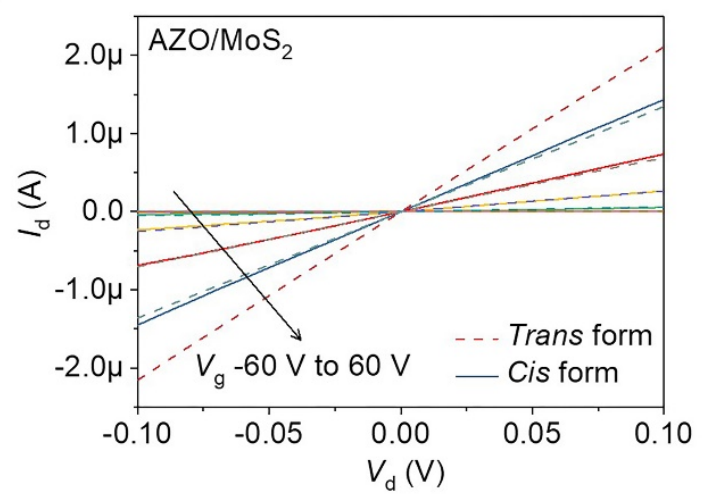

(e)

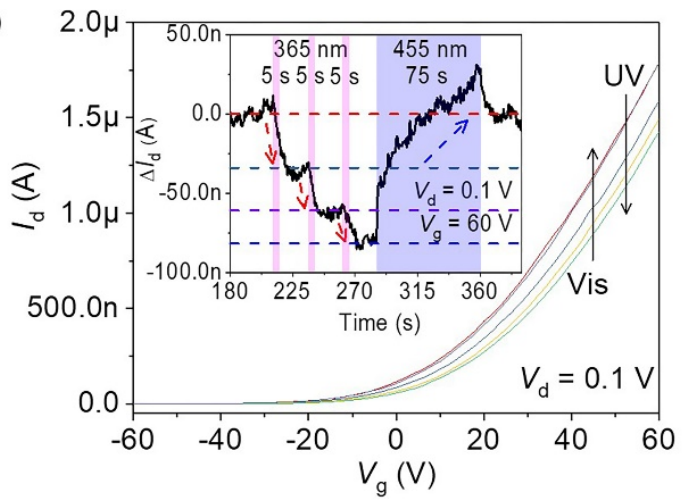

(b)

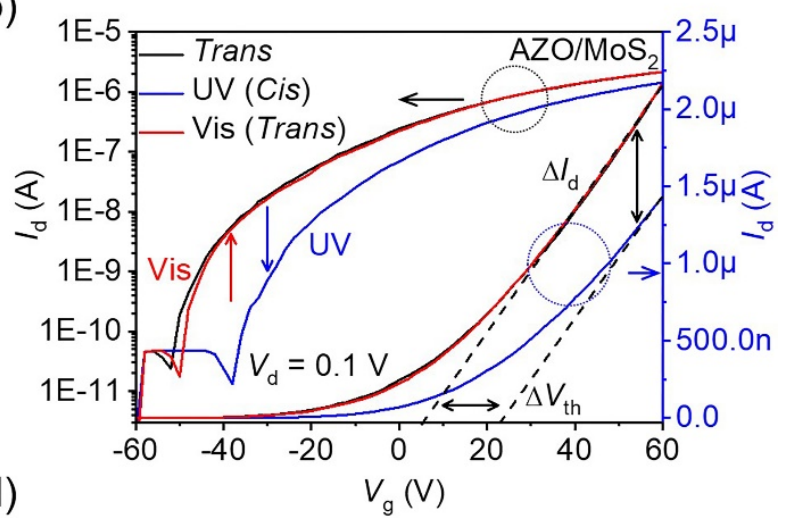

(d)

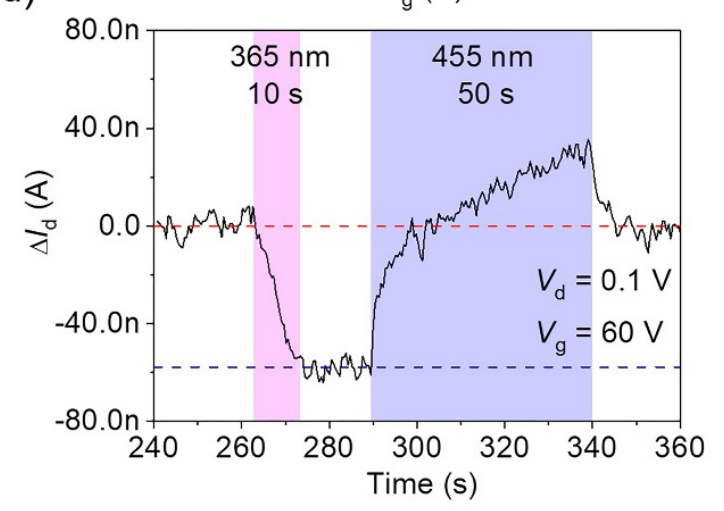

(f)

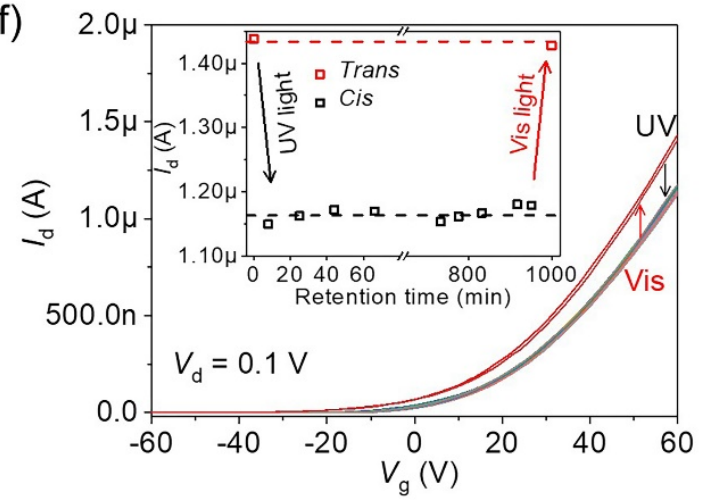

Figure 4. Electrical characteristics of $\mathrm{AZO} / \mathrm{MoS}_{2}$ hybrid devices. (a) Scheme of the hybrid FET structure with the physisorption of AZO molecules on $\mathrm{MoS}_{2}$ surface. (b) Transfer curves of the hybrid $\mathrm{AZO} / \mathrm{MoS}_{2}$ FET for one switching cycle (trans-cis-trans transition). (c) Output curves of the hybrid $\mathrm{AZO} / \mathrm{MoS}_{2}$ FET with trans- (dashed lines) and cis- (solid lines) AZO molecules. (d) Time-resolved photoresponse of the hybrid $\mathrm{AZO} / \mathrm{MoS}_{2} \mathrm{FET}$ under UV and visible light irradiation for one cycle. (e) Transfer curves of the hybrid AZO/MoS 2 FET with different trans/cis ratio of AZO molecules. The molecules ratio is controlled by the UV irradiation time. Inset shows the time-resolved photoresponse of the hybrid $\mathrm{AZO} / \mathrm{MoS}_{2} \mathrm{FET}$ under short-time UV irradiation. Four current levels are achieved by different time of UV light irradiation and recovered to pristine level by long-time visible light irradiation. (f) Transfer curves of the hybrid AZO/MoS 2 FET for the whole retention test. Inset shows the drain current at fixed $V_{\mathrm{g}}=60 \mathrm{~V}, V_{\mathrm{d}}=0.1 \mathrm{~V}$ after different time of storage in the dark to illustrate the retention capability of the device in the cis-AZO form. 

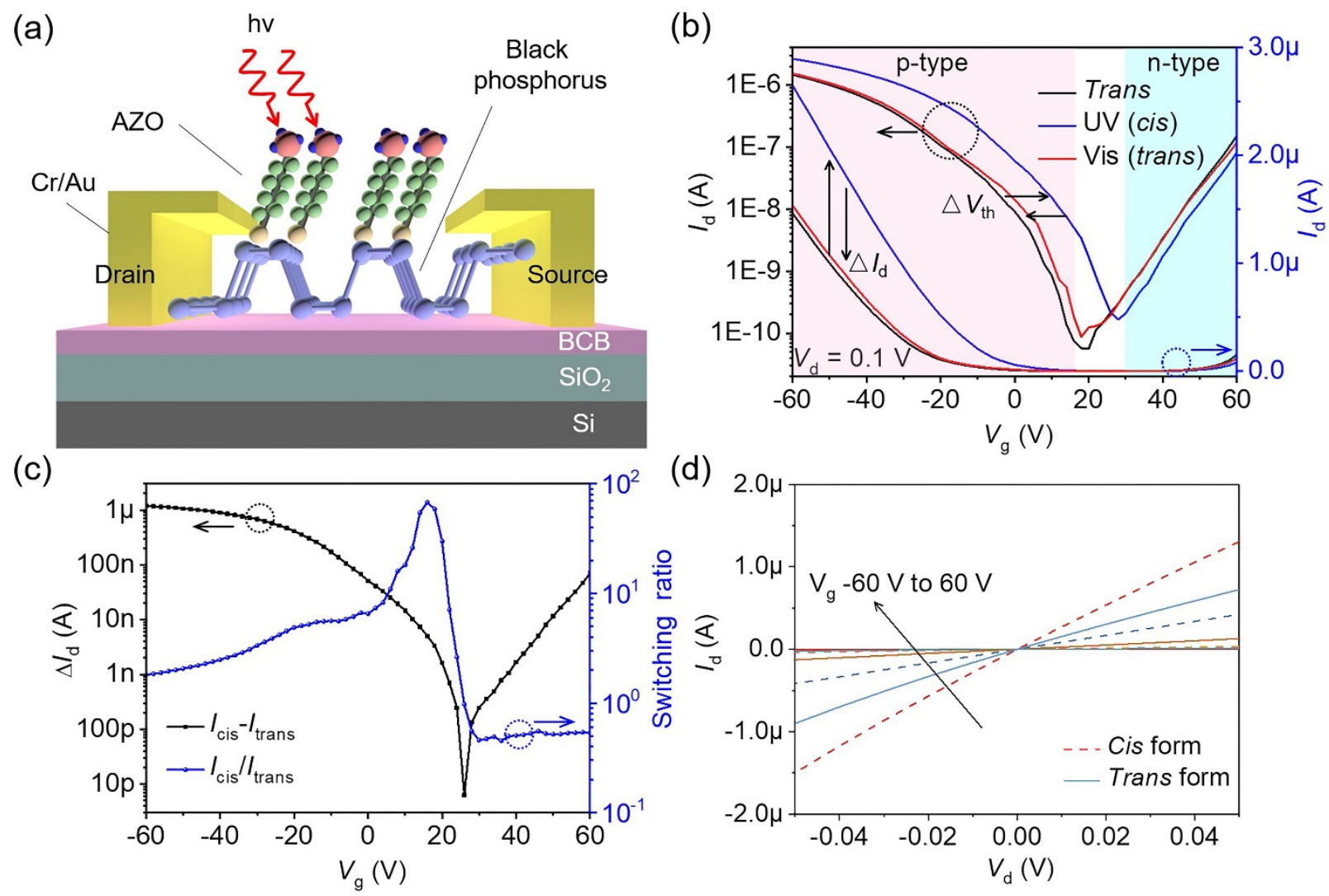

Figure 5. Electrical characteristics of AZO/BP hybrid devices. (a) Scheme of the hybrid FET structure with the physisorption of AZO molecules on BP surface. (b) Transfer curves of the hybrid AZO/BP FET with trans- and cis-AZO adsorption. (c) Current change $\left(\Delta I_{\mathrm{d}}=I_{\text {ciss }} I_{\text {trans }}\right)$ and switching ratio $\left(I_{\text {cis }} / I_{\text {trans }}\right)$ of the hybrid AZO/BP FET with trans- and cis-AZO adsorption. (d) Output curves of the hybrid AZO/BP FET with trans- (solid lines) and cis- (dashed lines) AZO molecules. 
(a)

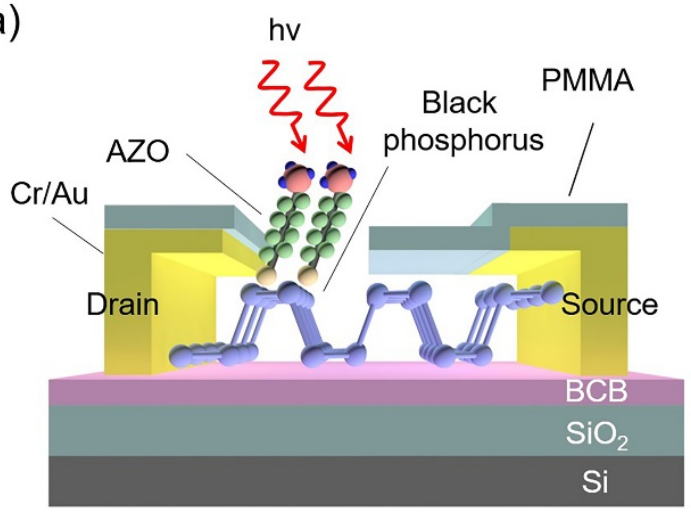

(c)

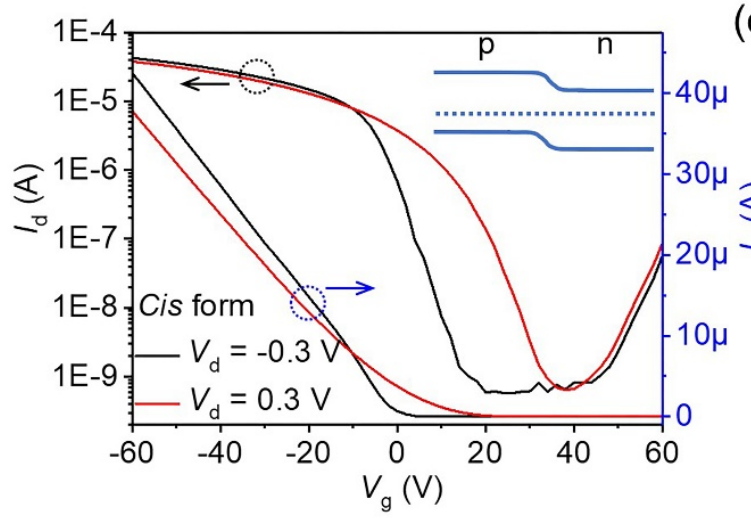

(b)

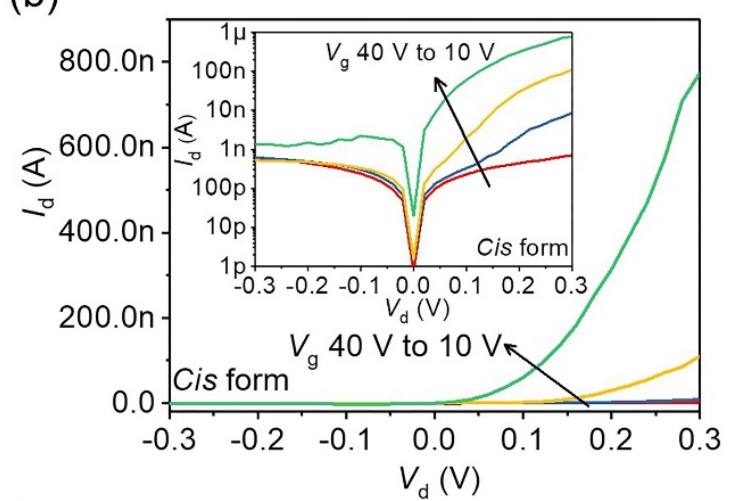

(d)

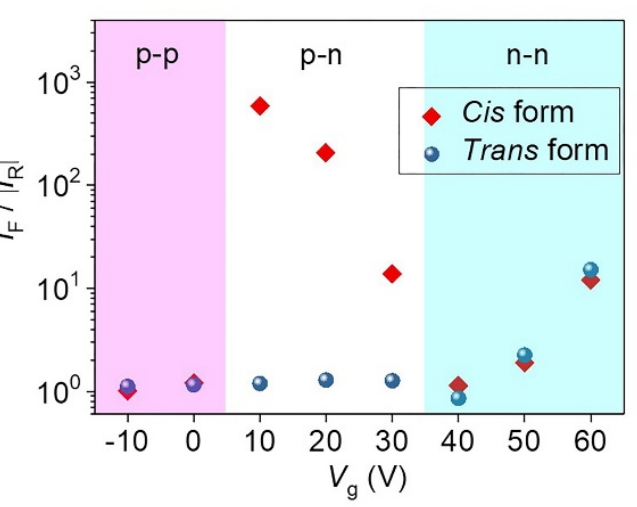

Figure 6. Electrical characteristics of BP based lateral heterojunction. (a) Scheme of the BP based lateral heterojunction with selective adsorption of AZO on patterned channel area. (b) Output curves of the patterned AZO/BP FET with cis-AZO adsorption. Inset shows the logarithmic plot of the output curves. (c) Transfer curves of the patterned AZO/BP FET with cis-AZO adsorption under $\pm 0.3 \mathrm{~V}$ drain bias. Inset shows the schematic energy-band diagram of the BP heterojunction when $V_{\mathrm{g}}$ is between $10 \mathrm{~V}$ and $30 \mathrm{~V}$. (d) Rectification ratio of the BP heterojunction under different molecular state and gate voltage. 